\title{
Electrodynamic sources of non-uniform heat production in composite superconductors causing instabilities*
}

\section{L.J.M. van de Klundert ${ }^{\dagger}$}

University of Twente, PO Box 217, 7500 AE Enschede, The Netherlands

Model calculations of the electrodynamic properties of superconducting composites are usually carried out assuming that the material constants, such as critical current density and resistivity, are uniform over the cross-section of the composite, or at least rotationally symmetric. Also, the applied field is usually considered to be uniform over a distance compared to the composite diameter. In this paper the possibility will be discussed that non-uniformity in field and material properties may cause significant non-uniformity in heat production so to disturb the local temperature pattern and form sources of instabilities and quenches. The influence of the cooling conditions on the transport current-carrying capacity in the a.c. regime is also discussed.

Keywords: composites; a.c. losses; transport current

\begin{tabular}{|llll|}
\hline \multicolumn{2}{|c|}{ Nomenclature (all units SI) } & $T_{\mathrm{f}}$ & Temperature in filamentary zone \\
$r, \varphi, z$ & Cylindrical coordinates & $T_{\max }$ & Maximum temperature in a cycle \\
$\partial_{r}, \partial_{\varphi}, \partial_{z}, \partial_{t}$ & Partial derivatives & $T_{\mathrm{s}}$ & Temperature in stabilizing material \\
$\mathbf{B}$ & Magnetic inducation vector & $T_{\text {start }}$ & Starting temperature \\
$B^{\mathrm{A}}$ & Applied perpendicular field & $\lambda$ & Electrical conductivity \\
$\mathbf{E}$ & Electric field vector & $\mathcal{B}$ & Thermal conductivity \\
$I^{\mathrm{A}}$ & Applied current amplitude & $\mathcal{E}$ & Value of $\mathbf{B}$ averaged over $\varphi$ \\
$\mathbf{j}$ & Current density vector & $\mathbf{E}$ averaged over $\varphi$ \\
$j_{\mathrm{c}}$ & Critical current density & $\mathrm{j}$ & Value of $\mathbf{j}$ averaged over $\varphi$ \\
$k$ & Applied field-to-current ratio & Subscripts & \\
$r_{1}$ & Inner radius of filamentary zone & $r$ & $r$-component \\
$r_{2}$ & Outer radius of filamentary zone & $r$ & $\varphi$-component \\
$R$ & Wire radius & $\varphi$ & $z$-component \\
$t$ & Time & $z$ & critical value \\
$T$ & Temperature & $\perp$ & component perpendicular to filament \\
$T_{\mathrm{c}}$ & Critical temperature & $\|$ & direction \\
$T_{\mathrm{e}}$ & Equilibrium temperature & & \\
$T_{\mathrm{b}}$ & Temperature of bath (heat sink) & & \\
\hline
\end{tabular}

The application of superconductors in the a.c. regime of transport current and applied fields, especially in the power frequency range $50-60 \mathrm{~Hz}$, encounters many difficulties. In principle, these are related to heat production and local increase of temperature, resulting in significant degradation of the transport current-carrying

* Paper presented at Second Joint Seminar (US - Japan) on 'Basic Mechanisms of Helium Heat Transfer and Related Influence of Superconducting Magnets', $26-30$ August 1991, Los Angeles, CA, USA.

${ }^{\dagger}$ Author deceased 12 September 1991; correspondence can be sent to H.J.J. ten Kate at the University of Twente.

$0011-2275 / 92 / 050508-05$

(c) 1992 Butterworth - Heinemann Ltd

508

Cryogenics 1992 Vol 32, No 5 capacity, sometimes amounting to $80 \%$. Even then, 'spontaneous' quenches occur far too often to allow application on a commercial and industrial basis in utility systems.

At least three sources of heat production can be identified. The first is the well-known electromagnetic power loss resulting from coupling and magnetization currents in the composite. Reduction of the filament diameter below $1 \mu \mathrm{m}$ and increase of the matrix resistivity by using $\mathrm{CuNi}$ largely reduces this amount of power loss but, in doing so, the thermal transport and mechanical properties also are significantly changed. Other sources of heat production result from the 
mechanical stability of the composite in the magnet. Due to the large Lorentz forces acting at double the frequency of the applied field and current, mechanical hysteresis may result in heat production. Finally, small wire motion and accompanying friction may release heat in unwanted catastrophic quantities.

In this paper we shall confine ourselves to the first source of electrodynamic heat production and the resulting temperature profiles in the conductor. The problem of isothermal heat production has been the subject of numerous studies in the past decades and it may seem that there is not much that can be added to it. Since, however, there is too large a discrepancy between established model calculations and experimental results in cases where mechanical sources of instability have to be excluded, a careful review of the established models was undertaken, and the results are presented below.

As a guideline for our research we took the rule that local excessive heat production in a wire may cause unexpected quenching and as a consequence the influence of local variations in material constants due to the production process, or local variations in the nonuniformity of the applied field, have to be studied.

\section{Transport equations in the filamentary region}

The cross-section of the composite superconducting wire, with radius $R$, consists of a ring-shaped filamentary zone, $r_{1} \leq r \leq r_{2}$, and an inner and outer region of normal conducting, stabilizing material. The area fraction $\eta_{\mathrm{f}}$ occupied by the filamentary zone is given by

$\eta_{\mathrm{f}}=\left(r_{2}^{2}-r_{1}^{2}\right) / R^{2}$

If $\eta_{\mathrm{sc}}$ is the volume fraction of superconductor in the whole wire the local fraction $\eta$ of superconductor in the filamentary zone is then given by

$\eta=\eta_{\mathrm{sc}} / \eta_{\mathrm{f}}$

Typical values of $\eta_{\mathrm{sc}}$ range from 0.1 to 0.4 , whereas $\eta$ normally is larger than 0.5 . The layout of the wire is shown in Figure 1. The effective values of $\lambda_{\|}$and $\sigma_{\|}$, the thermal and electrical conductivity parallel to filament direction respectively, are given by

$\lambda_{\sharp}=(1-\eta) \lambda_{0} \quad$ and $\quad \sigma_{\|}=(1-\eta) \sigma_{0}$

$\lambda_{0}$ and $\sigma_{0}$ are the isotropic bulk values of the matrix material. For the direction perpendicular to filament axis

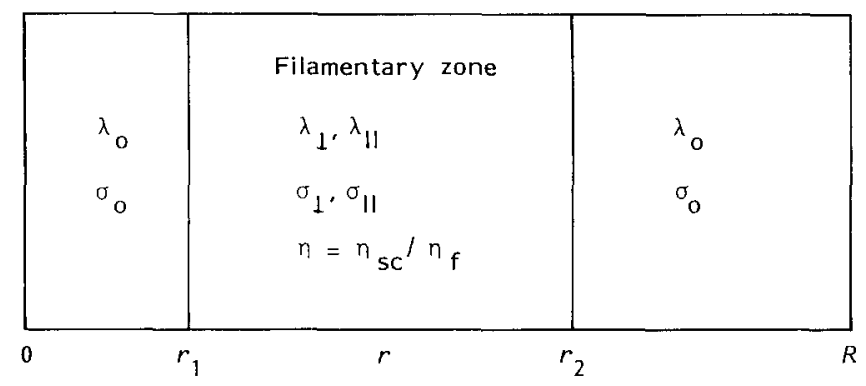

Figure 1 Layout of the wire
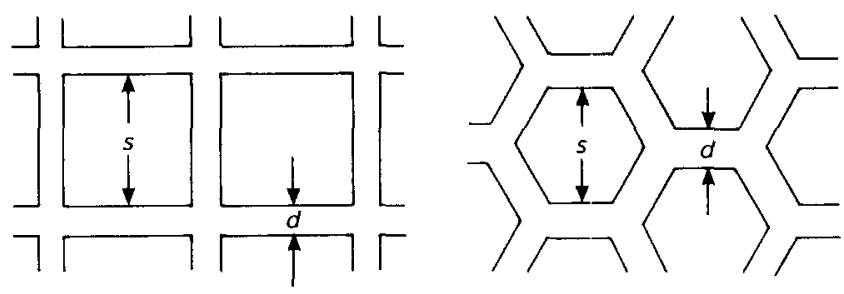

Figure 2 Arrangement of filaments in four-and six-fold symmetry

only coarse-grained isotropic values of $\lambda_{\perp}$ and $\sigma_{\perp}$ exist if the arrangement of filaments has a four- or six-fold symmetry. Then

$\lambda_{\perp}=\frac{1-\eta}{1+\eta} \lambda_{0}, \quad \sigma_{\perp}=\frac{1-\eta}{1+\eta} \sigma_{0}$

if we assume that the filaments do not participate in the transport process, that is, they have to be considered as vacuum. The factor $(1-\eta) /(1+\eta)$ is a good approximation for $\eta<0.6$ for filaments of arbitrary crosssection and both symmetries. For round filaments and four-fold symmetry at $\eta=\pi / 4=0.79$, however, $\lambda_{\perp}=0$, since the filaments will touch each other and no conducting path will remain.

In six-fold symmetry $\lambda_{\perp}$ will vanish at $\eta=\pi / 2 \sqrt{3}=0.91$. The function $\sigma_{\perp}(\eta)$ has been investigated for values of $\eta$ close to $\pi / 2 \sqrt{3}$ by Kanbara ${ }^{1}$ and Rem $^{2}$. In modern production technology, however, NbTi rods with matrix material are hexagonized before stacking them into billets. So the final filaments also have a more or less hexagonal cross-section. Now $\sigma_{\perp}(\eta)$ is non-zero for all $\eta<1$. For $\eta$ close to 1 in this case

$\sigma_{\perp}(\eta)=2(1-\sqrt{ } \eta) /(1+\sqrt{ }) \sigma_{0}=\frac{2 d}{2 s+d} \sigma_{0}$

where $s$ and $d$ are explained in Figure 2.

For square filaments in a four-fold symmetry the same approximation holds, which is close to the general $\eta$ dependence given in Equation (2). Detailed investigations on the behaviour of $\sigma_{\perp}(\eta)$, and consequently $\lambda_{\perp}(\eta)$, will be published elsewhere ${ }^{3}$. As a consequence, the thermal and electrical conductivities are reduced by one order of magnitude if $\eta=0.8$. This reduces the coupling losses, but a steep temperature profile may be built up in the wire. The loss reduction may be even larger when $\mathrm{Cu} 0.5 \% \mathrm{Mn}$ alloy is used in order to suppress the proximity coupling, as proposed for the SSC conductor ${ }^{4}$ at the expense of worse thermal conditions.

\section{The heat balance equation}

The heat balance equation in cylindrical coordinates in the filament region can be derived from the thermal transport equations

$C \frac{\partial T}{\partial t}=\operatorname{div}(\overline{\bar{\lambda}} \operatorname{grad} T)+p$ 
where $p=\mathbf{j} \cdot \mathbf{E}-\mathbf{M} \cdot \dot{\mathbf{B}}$ is the local loss power density $^{2}, \mathbf{j}$ is the current density; $\mathbf{E}$ the electric field; $\mathbf{M}$ the magnetization and $\dot{\mathbf{B}}$ the field rate.

Due to twist of the filaments, with twist length $L_{\mathrm{p}}$, the term $\operatorname{div}\left(\bar{\lambda} \operatorname{grad} T_{\mathrm{t}}\right)$ in the filamentary zone can be written as ${ }^{5}$

$$
\begin{aligned}
& \frac{1}{r} \partial_{r} \lambda_{r} r \partial_{r} T_{\mathrm{f}}+\frac{1}{r^{2}} \partial_{\varphi} \lambda_{\varphi \varphi} \partial_{\varphi} T_{\mathrm{f}}+\frac{1}{r} \partial_{\varphi} \lambda_{\varphi z} \partial_{z} T_{\mathrm{f}} \\
& \quad+\frac{1}{r} \partial_{z} \lambda_{\varphi z} \partial_{\varphi} T_{\mathrm{f}}+\partial_{z} \lambda_{z z} \partial_{z} T_{\mathrm{f}}
\end{aligned}
$$

with

$\lambda_{r}=\lambda_{\perp} \quad \lambda_{\varphi z}=\left(\lambda_{\|}-\lambda_{\perp}\right) \sin \psi \cos \psi$ $\lambda_{\varphi \varphi}=\lambda_{\perp} \cos ^{2} \psi+\lambda_{\|} \sin ^{2} \psi \lambda_{z z}=\lambda_{\perp} \sin ^{2} \psi+\lambda_{\|} \cos ^{2} \psi$

and the twist angle $\psi=\arctan \left(\pi r / L_{\mathrm{p}}\right)$

In the untwisted stabilizing matrix material $\operatorname{div}\left(\lambda_{0}\right.$ $\operatorname{grad} T_{\mathrm{s}}$ ) reads

$\frac{1}{r} \partial_{r} \lambda_{0} r \partial_{r} T_{\mathrm{s}}+\frac{1}{r^{2}} \partial_{\varphi} \lambda_{0} \partial_{\varphi} T_{\mathrm{s}}+\partial_{z} \lambda_{0} \partial_{z} T_{\mathrm{s}}$

The boundary conditions read

$$
\begin{array}{ll}
\lambda_{0} \partial_{r} T=h(\varphi)\left(T-T_{0}\right) & \text { at } r=R \\
\partial_{r} T(r, \varphi)+\partial_{r} T(r, \varphi+\pi)=0 & \text { at } r=0 \\
T(r, \varphi, \pm L)=T_{0} & L \gg R
\end{array}
$$

At the interface between filament region and stabilizing material

$$
\lambda_{\perp} \partial_{r} T_{\mathrm{f}}-\lambda_{0} \partial_{r} T_{\mathrm{s}}=0, r=r_{1}, r_{2} ; \quad \text { with } T_{\mathrm{f}}(r)=T_{\mathrm{s}}(r)
$$

It may be noted that in this description the temperature in the filament region $T_{\mathrm{f}}$ is the same for the superconductor as for the matrix. Collings ${ }^{6}$ reviews the possibility of temperature jumps at the superconducting filament-matrix interface. Using the above calculation method, therefore, too small an increase of the temperature $T_{\mathrm{f}}$ may be obtained.

\section{The loss power density $p(r, \varphi, z, t)$}

By analogy with the thermal transport equations the electric transport or constitutive equations can also be derived.

$j_{\perp}=\sigma_{\perp} E_{\perp}$

$j_{\|}=\eta j_{\mathrm{s}}+\sigma_{\|} E_{\Uparrow}$

Two expressions for $j_{s}$ may be considered, depending on whether we want to include the dimension for the filament or not. If we take $R_{\mathrm{f}} \neq 0$ and define

$E_{0}=\frac{8}{3 \pi} R_{\mathrm{f}}\left|\mathbf{B}_{\perp}\right|$ it has been shown ${ }^{2}$ that

$j_{\mathrm{s}}=j_{\mathrm{c}}\left(B_{\perp}, T\right) E_{\|} / E_{0}$, if $\left|E_{\|}\right|<E_{0}$

$j_{\mathrm{s}}=j_{\mathrm{c}}\left(B_{\perp}, T\right) \operatorname{sign}\left(E_{\|}\right)$, if $\left|E_{\|}\right| \geq E_{0}$

For $R_{\mathrm{f}}=0$, i.e. $E_{0}=0$, the values of $j_{\mathrm{s}}$ with $\left|j_{\mathrm{s}}\right|<j_{\mathrm{c}}$ will be determined by $\operatorname{div} \mathbf{j}=0$ (see later). The magnetization of round filaments carrying a transport currrent $j_{\mathrm{s}}$ can be approximated by

$M_{\perp}=(-4 / 3 \pi) \eta j_{\mathrm{c}} R_{\mathrm{f}}\left[1-\left(j_{\mathrm{s}} / j_{\mathrm{c}}\right)^{2}\right] \operatorname{sign}\left(\dot{B}_{\perp}\right)$

For filaments of different cross-section, minor adaptations have to be made, which can be neglected, especially when $j_{\mathrm{s}} / j_{\mathrm{c}}$ differs significantly from 0 . In cylindrical coordinates the constitutive equations now read

$j_{r}=\sigma_{\perp} E_{\mathrm{r}}$

$j_{\varphi}=j_{\mathrm{s}} \sin \psi+\sigma_{\varphi \varphi} E_{\varphi}+\sigma_{\varphi z} E_{z}$

$j_{z}=j_{\mathrm{s}} \cos \psi+\sigma_{\varphi z} Z_{z}+\sigma_{z z} E_{z}$

The dissipated power density is given by

$p=\mathbf{j} \cdot \mathbf{E}-\mathbf{M}_{\perp} \cdot \dot{\mathbf{B}}_{\perp}$

whenever the field change is larger than twice the penetration field of the filaments $B_{\mathrm{p}}=2 \mu_{0} j_{\mathrm{c}} R_{\mathrm{f}} / \pi$.

\section{The Maxwell equations}

In this section a simplified model will be given for the heat production in a wire with poorly conducting matrix $^{2,7,8}$. The purpose of the model is to calculate the major contributions to the loss power density, in this case the thermal transport current loss $j_{\|} E_{\|}$and the magnetization loss. Then, only $\varphi$ averages of the $\mathbf{E}, \mathbf{j}$ and $\mathbf{B}$ components have to be considered. Defining the average value $\mathbb{Q}$ of $\boldsymbol{A}$ by

$\mathcal{A}=(2 \pi)^{-1} \oint A \mathrm{~d} \varphi$

we get for the Maxwell equations, since $\varepsilon_{r}=B_{r}=0$ everywhere:

$$
\begin{aligned}
& \frac{1}{r} \partial_{r} r \mathcal{E}_{\varphi}=-\partial_{t} \Theta_{z} \quad \frac{1}{r} \partial_{r} r \Theta_{\varphi}=\mu_{0 j \|} \sin \psi \\
& \partial_{r} \mathcal{E}_{z}=\partial_{t} \Theta_{\varphi} \quad-\partial_{r} \Theta_{z}=\mu_{0 j \|} \cos \psi
\end{aligned}
$$

Taking $\varepsilon_{\|}=\varepsilon_{\varphi} \sin \psi+\varepsilon_{z} \cos \psi$ the constitutive equation reads

$j_{\|}=j_{\mathrm{c}}\left(B^{\mathrm{A}}, T\right) \mathcal{E}_{\|} / \mathcal{E}_{0}, \quad$ if $\quad\left|\mathcal{E}_{\|}\right|<\varepsilon_{0}$ $j_{\|}=j_{\mathrm{c}}\left(B^{\mathrm{A}}, T\right) \operatorname{sign}\left(\mathcal{E}_{\|}\right)$, otherwise; $\mathcal{E}_{0}=(8 / 3 \pi) R_{\mathrm{f}}\left|\dot{\leftrightarrow}_{\perp}\right|$

The boundary conditions are

$\mathcal{B}_{\varphi}\left(r_{2}, t\right)=\mu_{0} I^{\mathrm{A}} \sin \omega t /\left(2 \pi r_{2}\right), \mathcal{B}_{z}\left(r_{2}, t\right)=0$ 


$$
\mathrm{B}_{\varphi}\left(r_{1}, t\right)=0, \quad \mathcal{E}_{\varphi}\left(r_{1}, t\right)=-1 / 2 r_{1} \partial_{t} \mathcal{B}_{z}\left(r_{1}, t\right)
$$

We consider an applied field perpendicular to the wire axis and proportional to the transport current $B^{\mathrm{A}}(t)=$ $k I^{\mathrm{A}} \sin \omega t$.

For the critical current density a Kim relation is used, $j_{\mathrm{c}}(B, T)=j_{0} B_{0} /\left(B_{0}+|B|\right)$, where both $j_{0}$ and $B_{0}$ depend linearly on $T$.

$j_{0}=j_{00}\left(1-T / T_{\mathrm{c}}\right), B_{0}=B_{00}\left(1-T / T_{\mathrm{c}}\right)$

$j_{00}$ and $B_{00}$ are chosen in such a way that $j_{0}(4.2)=10^{10}$ A $\mathrm{mm}^{-2}, B_{0}(4.2)=2 \mathrm{~T}$ and $T_{\mathrm{c}}=9.2 \mathrm{~K}$.

The simultaneous solution of the heat balance and Maxwell equations leads to the results depicted in Figures 3 to 6 . At a low current amplitude, depending on the starting temperature $T_{\text {start }}$ and the heat sink temperature $T_{b}$, either the average temperature will increase depending on the heat production and cooling conditions, or, depending on the current amplitude, a temperature $T_{\max }$ in the wire will be reached twice in each period (see Figure 3).

Figure 4 shows, that depending on the amplitude, the wire will either quench or cool down again, when the starting temperature equals the bath temperature. In the

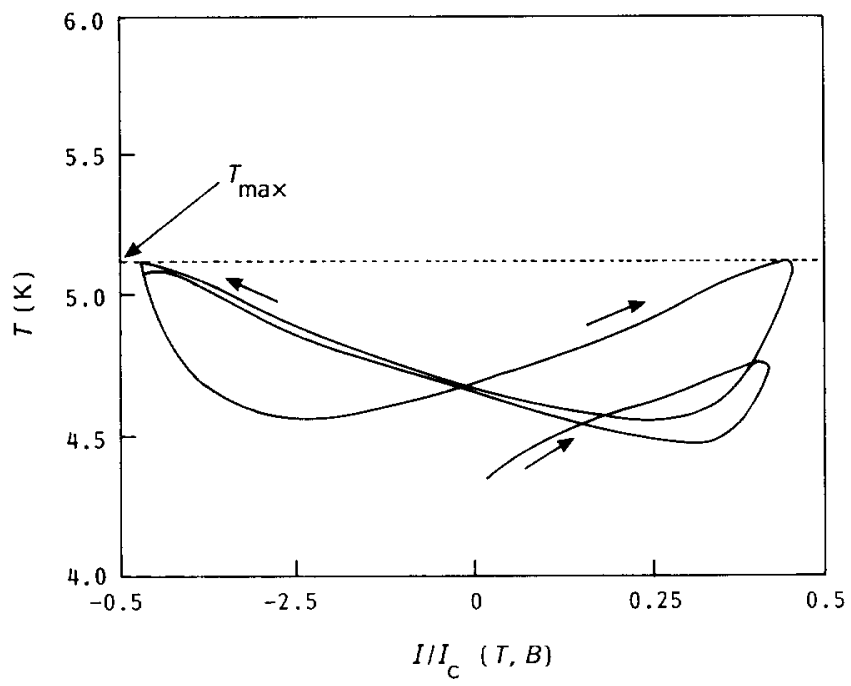

Figure $3 T$ vs / loop of the conductor, during several cycles

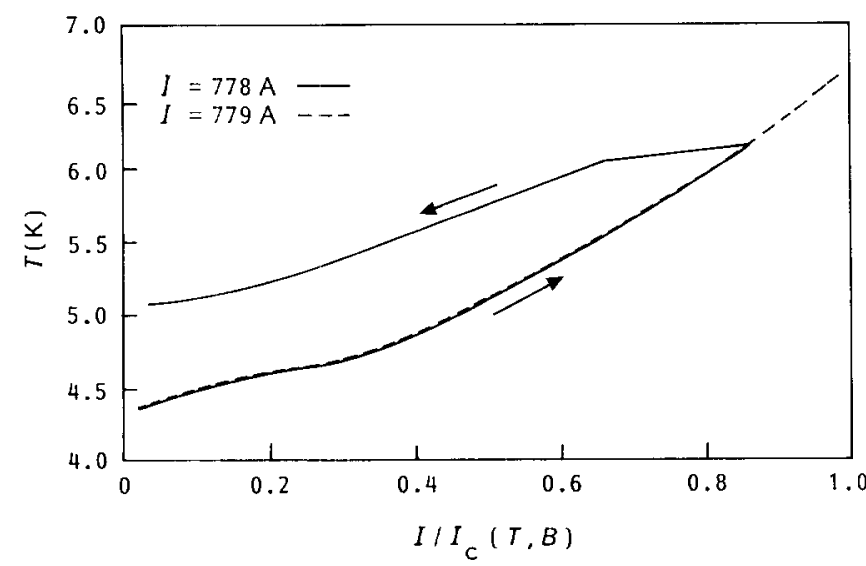

Figure $4 T$ vs / curves at two values of the amplitude, one quenching $(779 \mathrm{~A})$ and one non-quenching $(778 \mathrm{~A})$
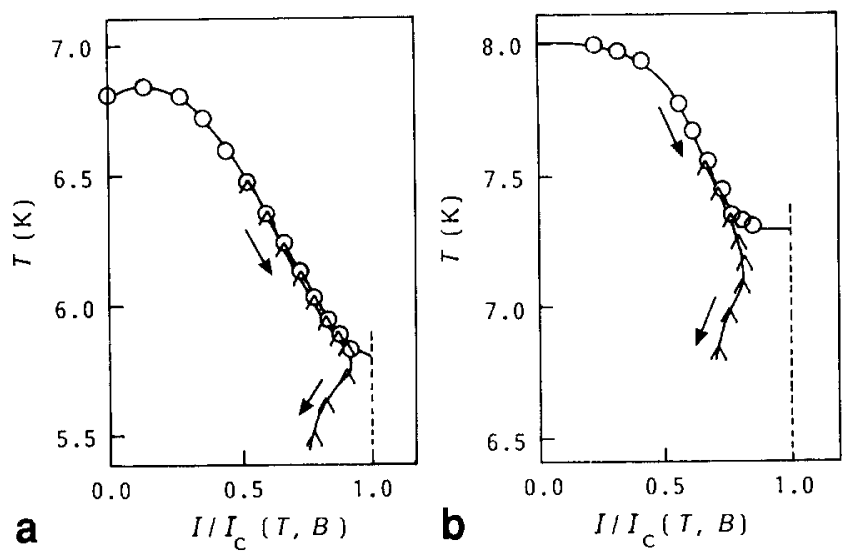

Flgure $5 T$ vs $/$ trajectories for a high starting temperature (a) $k=1 \mathrm{mT} \mathrm{A}^{-1}$; (b) $k=10 \mathrm{mT} \mathrm{A}^{-1} \cdot 1^{\mathrm{A}}=600 \mathrm{~A}$

same way, for each starting temperature $T_{\text {start }}>T_{\mathrm{b}}$ a current amplitude can be determined such that during cooldown the critical current $I_{\mathrm{c}}(T, B)$ is reached or not; see Figure 5 for such examples.

Figure 6 compiles the data for two values of $k, 1$ and $10 \mathrm{mT} \mathrm{A}^{-1}$, concerning the maximum temperature $T_{\mathrm{c}}$ that will be reached during each cycle whenever the starting temperature is lower than the line indicated by $T_{\mathrm{q}}$. Note that the higher value of $k$ determines the behaviour of the whole coil.

Varying the amplitude of the transport current (and so the amplitude of the applied magnetic field) and the initial temperature of the conductor, two situations may occur:

1 If the initial temperature is suffiently low (at a certain current amplitude) there will be a state of equilibrium after a few cycles at which the average heat production equals the heat flow to the bath. The amplitude of the current is plotted against the maximum temperature in the conductor in the left branches of Figure 6 (indicated by $T_{\mathrm{c}}$ ).

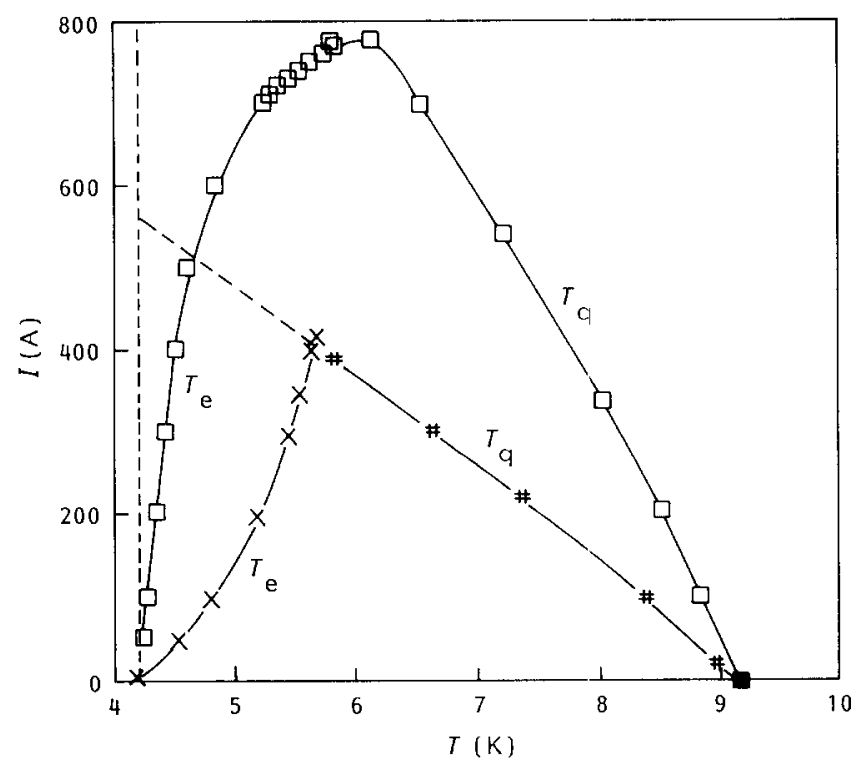

Figure 6 Stability diagram for two values of $k$ : $\square, 1 \mathrm{mT} \mathrm{A}^{-1}$; $\times$. $10 \mathrm{mT}^{-1}$. For starting temperatures smaller than $T_{\mathrm{a}}$ a stable situation with maximum temperature $T_{\mathrm{e}}$ will be reached 
2 If the initial temperature is too high, a quench will occur before the current will reach its maximum value. The current at which the wire quenches is plotted against the maximum temperature in the wire at the moment of the quench. These values are represented by the two right branches of Figure 6 (indicated by $T_{\mathrm{q}}$ ).

\section{Discussion}

In the above simplified example it was shown that large current reduction can be obtained due to the balance between heat production and cooling conditions. This balance can be disturbed in two ways: excessive heat production or reduced cooling. Excessive heat production may result from two sources: non-uniformity of the conductor layout and/or the local materials parameters, or non-uniformity of the applied field. The main source of non-uniformity in the conductor layout is the production process resulting in large local variations of the filament diameter. Variations up to a factor of two are possible. This results in low $n$ values because the transport current has to pass through the matrix when leaving the thin parts of the filaments. Also, the thickness of the interfilament layer may vary appreciably, but this is less significant for the local average values of the thermal and electrical conductivity. Nevertheless a global negative effect on the current-carrying capacity is expected.

The second part of the balance, the reduced cooling, seems more important. Particularly in twisted (multistage) cables the strands will touch each other tightly, in order to prevent wire motion, but at the same time thermal contact with the helium is strongly reduced. This is in accordance with experimental observations. Braided cables may have better cooling conditions, but lose some of their mechanical stability. Not only is heat production increased in wires subject to strongly inhomogeneous applied fields ${ }^{9}$, but also saturated regions are formed in the centre of the wire. Inhomogeneous applied field patterns may, for instance, result from current changes in neighbouring strands which are not cancelled by arguments of symmetry. In our model, saturated regions are responsible for high dissipation since the low electric matrix conductivity prevents part of the current sharing.

Consequently, cabled conductors may lose a part of their current-carrying capacity compared to the values obtained in experiments performed on well-cooled single-strand coils. It is suggested that experiments on non-inductively wound coils may provide additional information on the balance between electromagnetic heat production and cooling, since almost no mechanical instabilities are then expected due to the low Lorentz forces.

\section{Acknowledgements}

The author is very much indebted to A.J.M. Roovers and E.M.J. Niessen for useful discussions and assistance in performing the calculations.

\section{References}

1 Kanbara, K., Kubota, Y. and Ogasawara, T. Transverse field loss of a twisted multifilamentary round wire in windings of superconducting magnets Proc ICEC-9, Kobe 1982, Butterworths \& Co., Guildford (1983) 715

2 Rem, P.C. Numerical models for a.c. superconductors, Thesis, University of Twente (1986)

3 Niessen, E.M.J. and van de Klundert, L.J.M. The effect transverse resistivity in multifilamentary conductors, to be published

4 Spec. No. SSC Mag-M-4145/46, NbTi Superconducting wire for SSC dipole magnets, D. Capone, SSC-Lab. (1990)

5 Ishiyama, A., Sato, Y. and Tsuda, M. Normal zone propagation velocity in superconducting wires having a CuNi matrix IEEE Trans Magn (1991) 272076

6 Collings, E.W. Applied Superconductivity, Metallurgy and Physics of Titanium Alloys, Plenum Press, New York and London (1986) Vol. 1, $266-274$.

7 Roovers, A.J.M. and van de Klundert, L.J.M. Current distribution and a.c. losses in twisted multifilamentary a.c. superconductors IEEE Trans Magn (1988) 252127

8 Roovers, A.J.M. An experimental study of a.c. losses in superconducting wires and cables, Thesis, University of Twente (1989)

9 Niessen, E.M.J. and van de Klundert, L.J.M. Superconducting multifilamentary wires in inhomogeneous perpendicular a.c. fields Proc Int Symp AC Superconductors, Smolenice, CSFR (1991) 